\title{
Correspondence
}

\section{Audit of deaths from cervical cancer}

I read with interest the paper on audit of deaths from cervical cancer ${ }^{1}$ and concur with the approach the authors have taken.

This district will shortly be holding the fourth annual review of invasive cervical cancers, and has found the information gained from a multidisciplinary audit to be invaluable in identifying the "holes in the net" of the screening programme through which women fall.

The details of our audit were described at the annual Scientific Meeting of the British
Society for Clinical Cytology at Leeds in September $1993 .^{2}$ Our review differs in several small but important ways from that described by Slater $e t$ al.

We chose to widen the audit to include not only deaths from cervical cancer, but also non-fatal cases of invasive disease. All these women bear the burden of illness caused by cancer and its treatment. Inaccuracies in death certification and consequent problems with OPCS mortality statistics are well described. We have found that computed laboratory records are a more accurate and easily accessible method

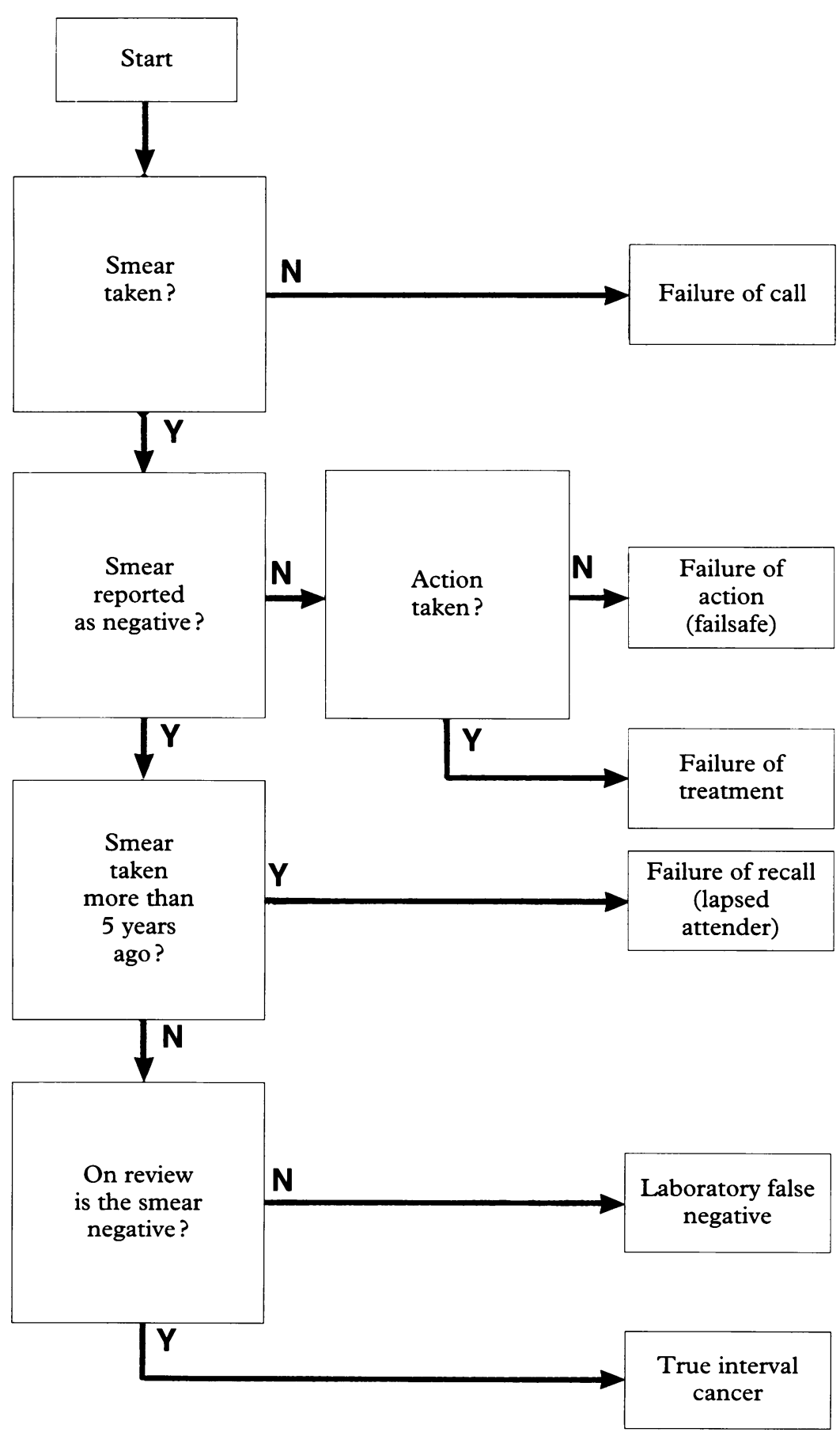

of identifying all cases. Moreover, only by reviewing all cases can one monitor one's performance against the Health of the Nation target, which calls for a reduction of $20 \%$ of cases of (not deaths from) invasive cervical cancer.

The method of reviewing previous smears and assigning cases to "true interval" or "false negative" categories is crucial and is obviously subject to enormous bias in the operation of the retrospectoscope. We have attempted to reduce any such bias by reviewing all smears reported as negative taken within five years of the onset of invasive disease and mixing them with a number (unknown to the reviewer) of true negative smears (reported as negative, with a subsequent normal smear at least three years later). It is vital that those reviewing smears should, as much as possible, be blind to the outcome following these smears. This manoeuvre encourages a more realistic approach to reviewing smears, so that any tendency to "overcalling" is minimised.

Slater et al do not describe the method used to assign deaths to each particular category. Our work in north Lincolnshire has led to the development of a simple algorithm (figure) by which all cases of cervical carcinoma are assigned to one (and only one) category. Although each centre could choose to perform its own particular form of audit, I believe a valuable opportunity will have been missed if differing forms of local audit proliferate. Valid comparisons between districts (and laboratories) will require standardised methods of defining these points of breakdown in the screening programme. The paper from Rotherham will, I hope, concentrate the minds of those responsible for national guidance in the operation of the cervical screening programme in setting up a useful system of audit throughout the country. ogy Department, County Hospital, Greetwell Road, Lincoln
LN2 $5 Q Y$

1 Slater DN, Milner PC, Radley H. Audit of deaths from cervical cancer: Proposal for an essential component of the National Screening Programme. f Clin Pathol 1994 47:27-8.

2 Hunt CM, Lamb MP, Breeson AJ, et al. Cervical screening: checking effectiveness by auditing "System Failures". Cytopathology 1993;4 Suppl 1:5.

\section{Cervical screening}

Two years ago a workshop of the National Co-ordinating Network (NCN) of the National Cervical Screening Programme issued guidelines for clinical practice which stated that, "smears showing viral changes but no nuclear change should be classified as normal." Now a working party of the NCN has issued guidelines, stating "that no cell with HPV infection is normal and that no smear in which there is evidence of HPV infection should be reported as negative." 2 I would be grateful if those members that served on both committees could explain why they have changed their advice.

A RUBIN

Department of Histopathology, Watford General Hospital, Vicarage Road, Watford, 
1 National Co-ordinating Network Workshop. Guidelines for clinical practice and programme management. 1992

2 NCN, BSCC and RCPath Working Party. Borderline nuclear changes in cervical management. f Clin Pathol 1994;47:481-92.

Dr Buckley (Chair) comments:

The Guidelines for Clinical Practice (NCN Workshop, 1992) did indeed state that "smears showing viral changes but no nuclear change should be classified as normal". The crucial point is that only when there are no nuclear changes should the cell be called normal. As only one member of the second working party (Joint Working Party, 1994) was able to produce a single example of a cell with viral (HPV) infection and a virtually normal looking nucleus, the members of the working party felt that the advice should be changed. Some members of the second working party would also like to make it clear that they disagreed quite firmly with the statement in the first document as it implied that this was a usual occurrence rather than a rarity.

\section{I read with interest the paper by ...}

Four of the six letters to the Editor in the April issue of the Journal begin with the statement "I (we) read with interest the paper by ..." (IRWITPB). One might have assumed that the preparation of a letter was sufficient evidence of an interest, stimulated in turn by reading the relevant paper. So why are they telling us of their interest?

Clearly many writers have information which supplements or clarifies an aspect of a paper or which corrects an error but there may be some additional factor, some intensity of interest which stimulates the letter, so, if subjective feelings are to be included, why stop at the bland "interest"? Why not indicate the author's true feelings and begin, for example, with, "We read with mounting indignation the flawed paper by ....," or "We were devastated to see in print a study almost identical to our soonto-be-completed study", or "We were dumbfounded to find that our paper was not cited by ..." The "interest" of other writers might be a code concealing lyrical, cynical or even machiavellian approaches.

I looked over the most recent 16 issues of the Journal and found that almost $40 \%$ (22 of 56) of letters began with IRWITPB or its variants (one writer was very interested, another fascinated; one simply said he very much enjoyed the paper). A warning to IRWITPB writers is the response to one letter, [they] may have read our article "with interest", but clearly they have not read it with "great care". The prevalence of IRWITPB indicates that rather than hiding an undisclosed intensity of interest, it has become a cliché to introduce a letter and, as such, should be discouraged ... or is anyone interested?

DS O'BRIAIN Histopathology Departmen St fames's Hospital, Dublin 8
Ireland
Benign lymph node inclusions mimicking metastatic carcinoma

Although previously well recognised, Fisher et al have highlighted the importance of differentiating between metastatic carcinoma and benign intranodal epithelial inclusions. I describe three further cases encountered recently in which immunocytochemical analysis provided valuable evidence to help clarify the nature of intranodal inclusions. In each case the differentiation was clinically critical in that no other nodes were involved.

\section{Case 1}

A 66 year old woman had a mass in the left breast detected by screening. A localisation biopsy specimen showed a grade 1 ductal carcinoma and a diffusely infiltrating lobular carcinoma. She underwent mastectomy: there was residual invasive lobular carcinoma and multiquadrant foci of invasive lobular carcinoma up to $7 \mathrm{~mm}$ diameter. The original tumour was estimated at 200 $\mathrm{mm}$ diameter and there was pagetoid spread of lobular carcinoma in ducts and extensive vascular invasion. Three of 12 axillary lymph nodes contained groups of small cells in the capsule. Initially thought to be metastatic lobular carcinoma, immunocytochemistry was performed to look for lesser degrees of spread to the lymph nodes in the same slide. Immunocytochemistry was performed on the breast tumour and the lymph nodes for HMFG-2 AE1/AE3, CAM 5.2, and $\mathrm{S} 100$. The lobular carcinoma stained strongly positively for AE1/AE3, CAM 5.2, and HMFG 2, and weakly for S100. Cells in the lymph node stained strongly for $\mathrm{S} 100$ but were otherwise negative, indicating that they were benign naevus cells. Interestingly, there were numerous $\mathrm{S} 100$ positive cells scattered through the lymph node parenchyma.

Case 2

A 65 year old woman had a cytologically malignant lump detected by screening in
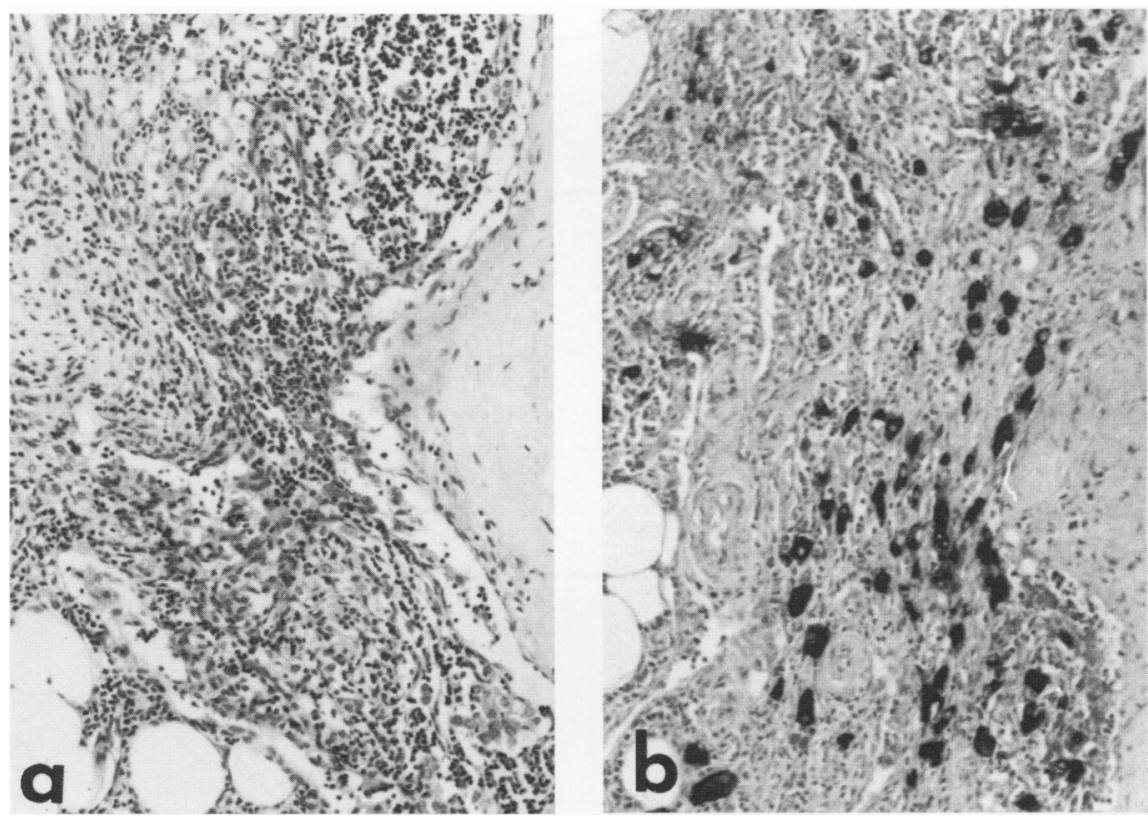

the left breast. She underwent mastectomy and histological examination showed a 14 $\mathrm{mm}$ lobular carcinoma. One of 17 axillary lymph nodes contained a suspicious focus of sclerosis and scattered histiocyte-like cells with clear cytoplasm. Periodic acid Schiff stain showed intracytoplasmic mucin, and immunocytochemical staining showed positivity for HMFG-2 and CAM 5.2 and was negative for carcinoembryonic antigen (CEA) in both intranodal cells and the original carcinoma, confirming metastatic lobular carcinoma (fig 1).

\section{Case 3}

A 26 year old woman had bilateral papillary serous cystodenomas of the ovaries of borderline malignancy excised. No unequivocal stromal invasion was seen, but tumour was present on the surface of the left ovary. The right external iliac lymph node was excised. Histological analysis showed a single large lymph node in which there were multiple subcapsular epithelial structures, some of which showed papillary structure with psammoma body formation. These were thought to be benign inclusions of mullarian type epithelium (fig 2). In an attempt to demonstrate disparity in immunocytochemical profile to support this diagnosis immunocytochemistry was performed on both ovarian tumours and the lymph node for CEA, vimentin, HMFG-2, S100, CAM $5 \cdot 2$ and AE1/AE3. The immunocytochemical markers were similar in the ovarian tumours and inclusions except that CA125 showed delicate membrane staining in both ovarian tumours but the epithelial structures in the lymph nodes did not stain.

In each of the cases described the lymph node diagnosis made the difference between lymph node positive and lymph node negative, with subsequent implications for clinical management. The cases illustrate the use of immunocytochemistry to support or refute a morphologically based opinion. The first case showed that naevus cell inclusions can line up in a pseudo-Indian file looking remarkably like metastatic lobular

Figure 1 Case 2: suspicious area in an axillary lymph node (a) haematoxylin and eosin;

(b) immunocytochemistry of same area showing numerous single HMFG-2 positive cells (metastatic lobular carcinoma). 